\title{
Wavelet, Gabor Filters and Co-occurrence Matrix for Palmprint Verification
}

\author{
Anouar Ben Khalifa \\ Research Unit of Advanced Systems in Electrical Engineering, \\ National Engineering School of Sousse, Tunisia \\ ben_khalifa_anouar@yahoo.fr \\ Lamia Rzouga \\ Research Unit of Advanced Systems in Electrical Engineering, \\ National Engineering School of Sousse, Tunisia \\ lamiarzouga@yahoo.fr \\ Najoua Essoukri BenAmara \\ Research Unit of Advanced Systems in Electrical Engineering, \\ National Engineering School of Sousse, Tunisia \\ najoua.benamara@eniso.rnu.tn
}

\begin{abstract}
Authentication through the palmprint is a field of biometrics. Palmprint-based personal verification has quickly entered the biometric family. It has become increasingly popular in the recent years due to its ease of acquisition, reliability and high user acceptance. In this paper, we present an authentication system based on the palmprint. We are particularly interested in the feature extraction step. Three feature extraction techniques based on the discrete wavelet transform, the Gabor filters and the co-occurrence matrix are evaluated. The support vector machine is used for the classification step. The results have been validated on the PolyU database related to 400 users. The best results have been achieved with the wavelet decomposition.
\end{abstract}

Index Terms - Biomtrics, Palmprint verification, PolyU database, support vector machines, wavelet, Gabor filters, co-occurrence matrix

\section{INTRODUCTION}

The authentication of people by the palmprint is a relatively new biometric technology compared to other modalities such as the signature, face or fingerprint. It has recently attracted the attention of many researchers. Several scientific researches in this area are underway. Fig. 1 shows the evolution of scientific productions on the palmprint biometrics in the IEEE journal. We find that the number of publications has increased by twentyfive times, within ten years, which demonstrates more scientific attention on this authentication technique.

Just like the fingerprint which is a unique signature for each person on the fingertips, the palm print is a unique signature to each individual defined on the palm of the hand. The palmprint can be acquired in a high- resolution or low-resolution mode (Fig. 2). The highresolution mode generally implies a resolution greater than or equal to 400 dpi $[4,17,26]$; it is commonly adopted in the case of forensic applications such as the dermatoglyphe and the detection of criminals where the information should be of a high quality [30]. In this mode, the biometric information is extracted from the ridges, singular points and minutiae points. The lowresolution mode generally implies a resolution less or equal to 150 dpi $[4,17]$ and it is more appropriate to civilian and commercial applications such as the access control to premises, the security of communication, the security of transacting information systems. In the lowresolution mode, the biometric information generally includes main lines which are generally clear and fine lines which are thinner and more irregular than the main lines and ridges scattered all over the palm [12, 18, 23, $24,31]$. Lines and wrinkles are formed between the third and fifth month of pregnancy while peaks appear after birth [26].

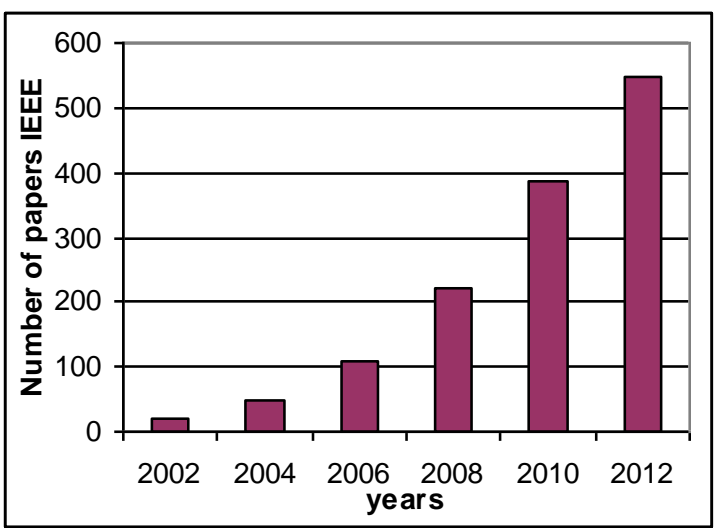

Figure 1. Evolution of the number of IEEE scientific publications on palmprint authentication 
We note that the majority of works dealing with the authentication of individuals by the palmprint uses lowresolution images because of its potential applications and low cost $[12,24,31]$.

The palmprint is a morphological biometric characteristic which is not genetically deterministic because it is different even for the identical twins [4, 30]. Biometrics by the palmprint has several advantages: the texture of the palmprint is richer than the fingerprint and so it is more discriminate [14, 27], the acquisition systems of the palmprint are less expensive than those of the iris. In addition, the palmprint is listed among the top four biometric characteristics that respond to the requirements of the biometric authentication systems since it appears as one of the less intrusive modalities, readily accepted by the public, and it does not require any effort or special cooperation of the user $[12,18,19$, $25,35]$. However, the authentication of individuals by the palmprint is not a trivial task. Indeed, the palm of the hand, like any biometric characteristic, is variable in time and sensitive to aging (body weight gain loss or). The acquisition conditions, such as lighting, bad positioning of the hand, dust on the sensor or humidity on the hand are also a constraint to the palm biometric [5]. Despite the significant progress in the recent years, the results are generally lower than those achieved in the case of the biometric authentication by the iris or the fingerprint, hence leaving the field open to other investigations.

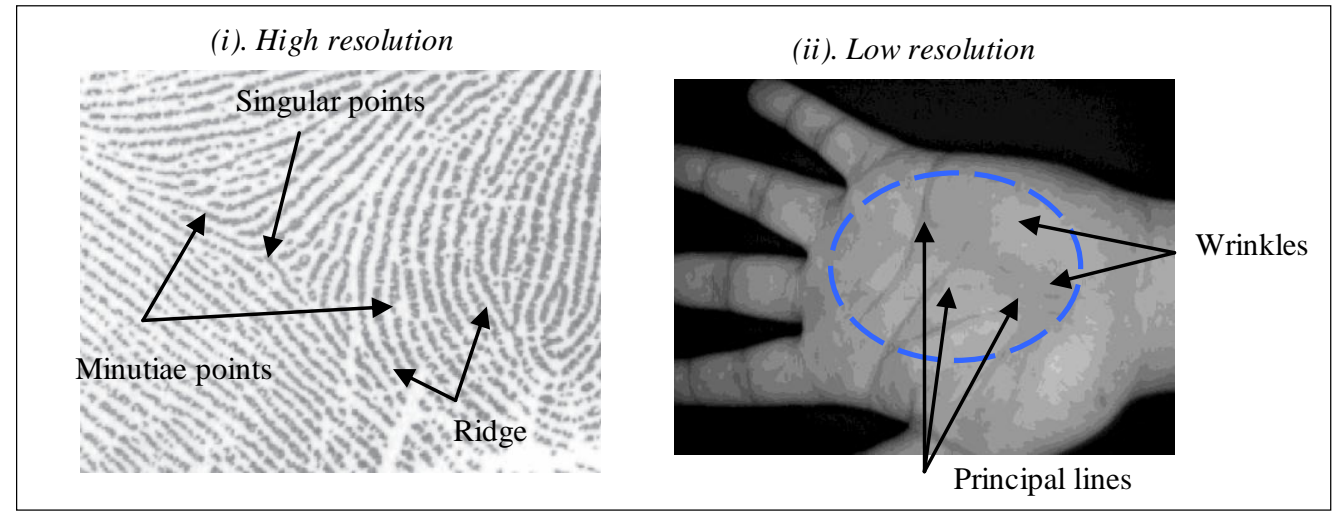

Figure 2. Palmprint acquired mode: (i) high resolution, (ii) low resolution.

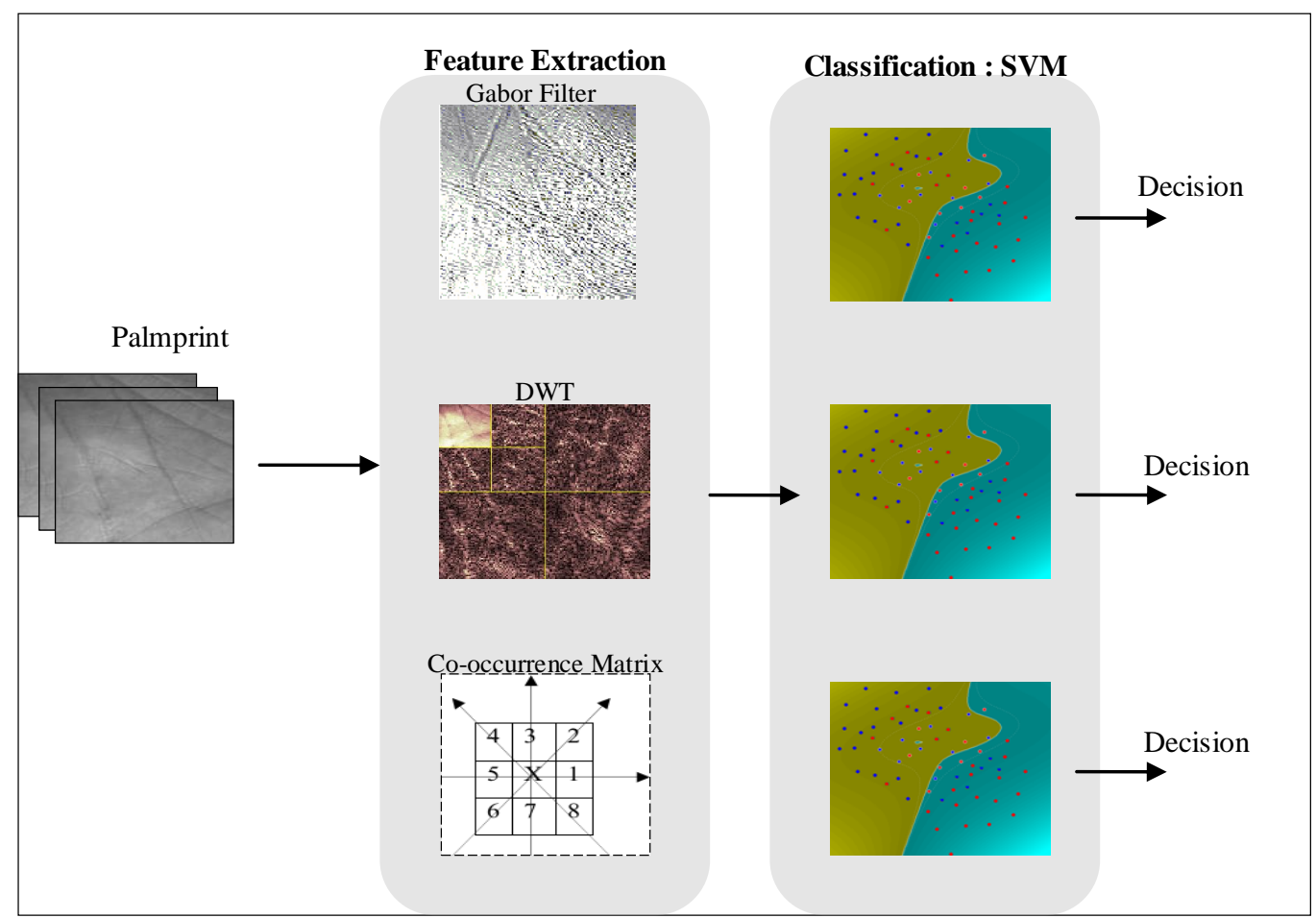

Figure 3. Overall architecture of the proposed system 
The literature reveals a variety of analysis approaches of the palmprint. Different feature extraction methods, geometric, structural, statistical, local, global and hybrid, have been exploited. Various classification techniques ranging from a simple Euclidean distance to the hybridization of classifiers have been validated $[4,5]$. In this paper, we propose a system of identity verification by the palmprint. This work falls within the framework of our research works in multimodal biometrics [1, 2, 3, 16]. We are particularly interested in the feature extraction step. Three approaches to the feature extraction based on the Discrete Wavelet Transform (DWT), the Gabor filters and the co-occurrence matrix are evaluated (Fig. 3). In the following, we give a report on the biometric authentication by the palmprint. In Section 3, we present the proposed approach. The obtained experiments and results are explained in section 4 .

\section{RELATED WORKS}

Several approaches for checking the identity of persons by the palmprint have been developed in the literature. These approaches can be classified as follows:

- Line-based approaches: In this approach, the identity verification is based on the comparison of the lines of the palm of the hand. Different methods have been used for the extraction of lines such as [6, 12, 18, 19, 27, 28]: Sobel masks, Canny filters, Hough transformation, histogram thresholding... The parameters adopted are generally the orientation of lines, their intersection points, their extremities...
- Subspace-based approaches: This approach is classically used in the context of face biometrics. In this approach, several linear or nonlinear decompositions are performed for modeling the skin of the hand palm. Among these approaches we mention $[12,15,17,20,32,34]$ : Principal Component Analysis (PCA), Independent Component Analysis (ICA), Linear Discriminant Analysis (LDA), Eigenpalms, Fisherpalms...

- Coding approaches: This approach exploits the richness of the texture of the palm in the frequency domain through the Fourier transform [19, 24, 33] or the time domain / frequency by the DWT [8, 20, 28, 31], the Discrete Cosine Transform (DCT) [11, $21]$ and the Gabor filters $[13,15,17,25,55]$.

- Statistical approaches: In this approach, we distinguish local and global statistical methods. The local statistical methods transform images in another area; these images are then divided into several regions (circular, square or elliptical). Statistical measurements such as the mean and standard deviation are calculated for each region and are considered as characteristics [22, 29]. The overall statistical methods operate on the whole image for calculating the features such as $[5,13,24]$ : Zernike moments, the variance, the center of gravity and density...

Table 1 presents a selection of works on the authentication of individuals by the palmprint.

Table 1: Summary table showing the characteristics and performance of some palmprint authentication systems. (EER: Equal Error Rate, RR: Recognition Rate)

\begin{tabular}{|c|c|c|c|c|}
\hline Ref. & Feature extraction & Classifier & database & $\begin{array}{c}\text { Performance } \\
(\%)\end{array}$ \\
\hline [29] & - Co-occurrence matrix & $\mathrm{k}-\mathrm{NN}$ & $\begin{array}{c}243 \text { users, } \\
1874 \text { images }\end{array}$ & $\mathrm{RR}=98.91$ \\
\hline [15] & $\begin{array}{l}- \text { PCA } \\
\cdot \text { DWT }\end{array}$ & Euclidean distance & $\begin{array}{l}386 \text { users, } 7752 \\
\text { images }\end{array}$ & $\mathrm{EER}=11$ \\
\hline [25] & - Gabor filters & Hamming distance & $\begin{array}{c}150 \text { users, } \\
900 \text { images }\end{array}$ & $\mathrm{EER}=10.3$ \\
\hline [18] & $\begin{array}{l}- \text { Lines of the palm } \\
\text { with the Canny filters }\end{array}$ & Hausdorff distance & $\begin{array}{c}386 \text { users, } 7752 \\
\text { images }\end{array}$ & $\mathrm{EER}=2.76$ \\
\hline [31] & - DWT & Neural network & $\begin{array}{c}7 \text { users } \\
70 \text { images }\end{array}$ & $\mathrm{RR}=97.14$ \\
\hline [20] & $\begin{array}{l}\text { - Contourlet tansforms } \\
\text { - Eigenpalms }\end{array}$ & Euclidean distance & $\begin{array}{c}24 \text { users } \\
120 \text { images }\end{array}$ & $\mathrm{RR}=94$ \\
\hline [22] & - Local binary patterns & $\mathrm{k}-\mathrm{NN}$ & $\begin{array}{c}146 \text { users } \\
1460 \text { images }\end{array}$ & $\mathrm{RR}=92.72$ \\
\hline [21] & - DCT & Canberra distance & $\begin{array}{c}50 \text { users } \\
500 \text { images }\end{array}$ & $\mathrm{RR}=97.5$ \\
\hline [12] & $\begin{array}{l}\text { - Gabor filters } \\
\text { - Principal lines } \\
\text { - Eigenpalms }\end{array}$ & $\begin{array}{l}\text { - Hamming distance } \\
\text { - Similarity measure } \\
\text { - k-NN }\end{array}$ & $\begin{array}{l}100 \text { users } \\
600 \text { images }\end{array}$ & $\begin{array}{l}\mathrm{RR}=84.3 \\
\mathrm{RR}=92.5 \\
\mathrm{RR}=95\end{array}$ \\
\hline [8] & - DWT & Euclidean distance & $\begin{array}{c}50 \text { users } \\
500 \text { images }\end{array}$ & $\mathrm{EER}=4.07$ \\
\hline
\end{tabular}


In what follows, we describe two works on the authentication of individuals by the palmprint. These works have been validated on the PolyU database [7], which we have used to validate our approach.

In [10], the authors present an authentication system by the palmprint. The system is based on analyzing the texture by the DWT. Several wavelet families have been involved; we mention: the DCT Transform Wavelet, the Haar Wavelet, the Slant Wavelet, the Hartley Wavelet... The method consists in calculating the Mean Square Error (MSE) between the reference images and the testing images resulting from the DWT. On the database relating to 8000 images, the recognition rate recorded is close to $93 \%$.

On the same database, Abeysundera et al. [9] present an approach invariant to rotation, and scaling: Scale Invariant Feature Transform. The features retained are the amplitudes and directions of this transformation. The classification is performed by the method of the $\mathrm{k}$ Nearest Neighbor (k-NN). The results achieved present a satisfaction in terms of False Acceptance Rate (FAR = $0.75 \%)$, and a low False Rejection Rate (FRR $=12.77 \%)$.

\section{THE PROPOSED FEATURE EXTRACTION APPROACHES}

The feature extraction is a very important step for the design of a biometric system. The objective of this step is to extract variables that describe, unequivocally, the forms belonging to the same class while differentiating them from the other classes. In our work, the proposed approaches are based on the texture analysis. Three techniques are compared: the Gabor filters, the DWT and the co-occurrence matrix.

Gabor filters: The Gabor filters are used to extract the relevant information, both in space and in frequency, relating to the texture. In our work and after various tests, we have retained the values of $30^{\circ}, 60^{\circ}, 120^{\circ}, 150^{\circ}$ orientations. For each orientation, different experimentations have led to choosing the three following frequencies: $\pi / 2, \pi$ and $2 \pi$ (Fig. 4). The mean and standard deviation of the filtered images have been selected as texture features. The feature vector is composed of 24 primitives associated with each filtered image.

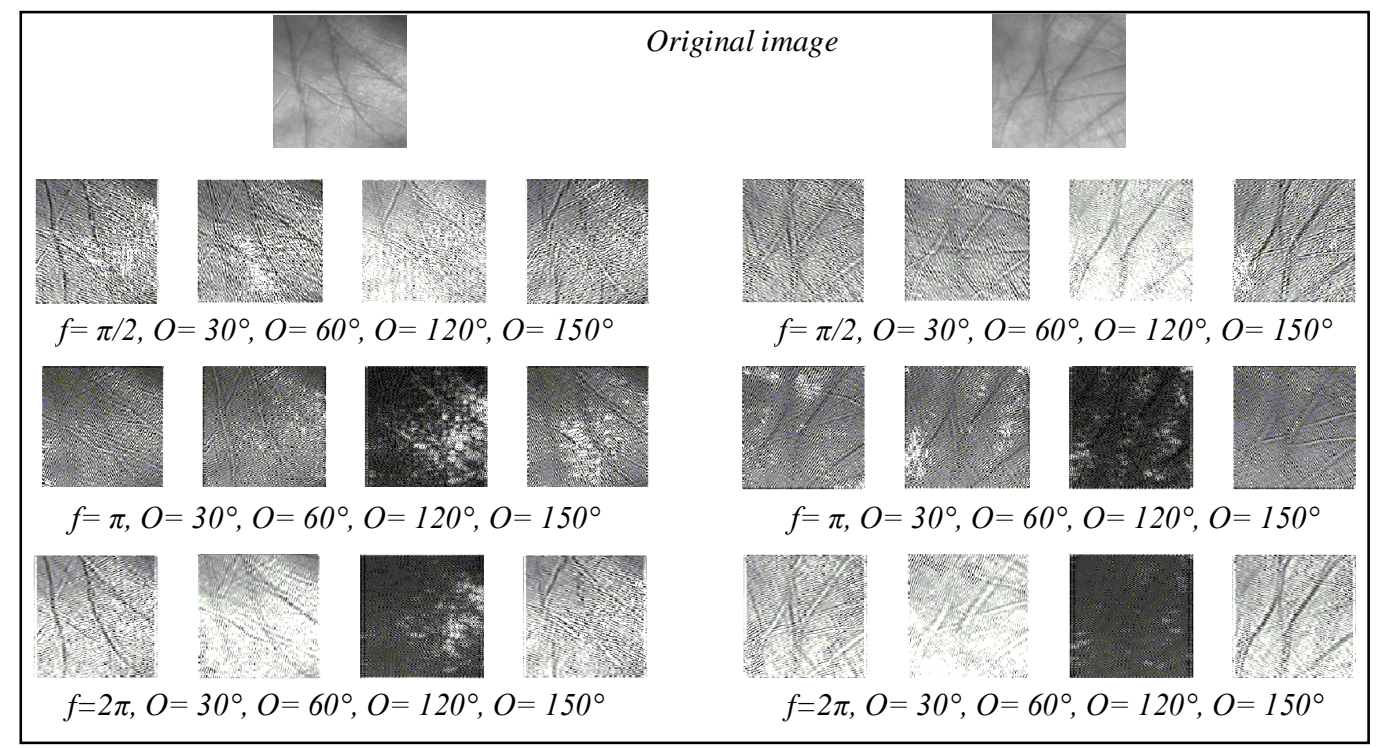

Figure 4. The images resulting from the Gabor filter for the used frequencies and orientations.

Discrete Wavelet Transform: The wavelet transformation has been widely used in various applications in image processing such as denoising, restoration and compression. It has proven its effectiveness especially in the texture analysis. Due to the multi-resolution principle, the DWT provides detailed information both in space and in frequency on the image, which allows analyzing texture at different scales. In our work and after various tests, we have used the Symlet6 at level 2 of the decomposition. The features used are the mean and standard deviation of the approximation matrix and the standard deviation of the horizontal, vertical and diagonal details. The images in Fig 5 show the DWT (Symlet6 at level 2) of two palmprint images.
Co-occurrence matrix: Because of its richness in texture information, the co-occurrence matrix has become one of the most widely used approaches to extract features of textures. The co-occurrence matrix measures the appearance probability of the pairs of the values of the pixels situated at some distance in the image. Several information can be extracted from the co-occurrence matrix. In our work, we have brought our selection on the four most salient parameters in the literature, namely: contrast, correlation, energy and homogeneity. 


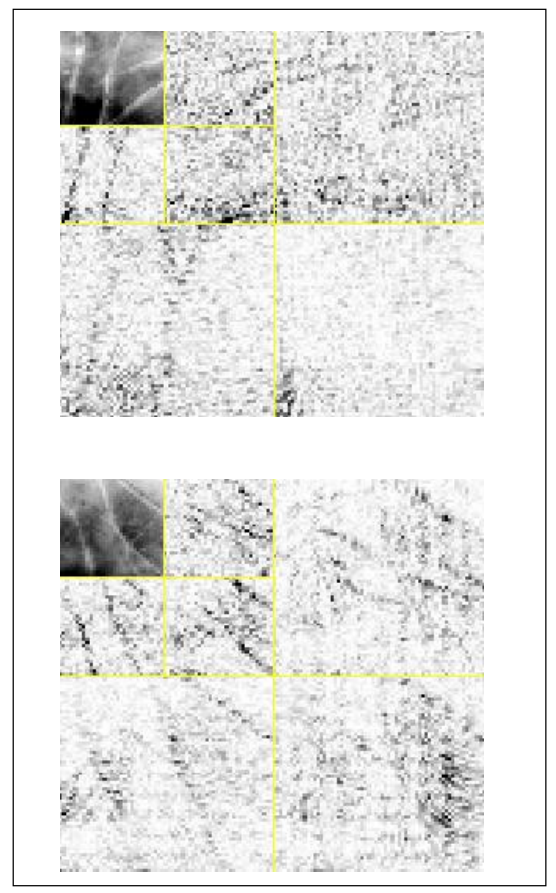

Figure 5. DWT (Symlet6 at level 2) of two palmprints.

\section{IV.EXPERIMENTATION AND RESULTS}

The experiments have been performed on an Intel Dual-Core PC, having $1.73 \mathrm{GHz}, 1 \mathrm{~GB}$ RAM, with the Matlab R2007 and Visual C++ environment under the Windows XP platform. To confirm the validity of the proposed approach, we have implemented the palmprint databases in PolyU. The palmprint images are obtained from the Hong Kong Polytechnic University 2D_3D palmprint database [7]. The database consists of 400 subjects with 20 palmprint images available for each subject. All palmprints are in greyscale images and a $128 \times 128$ resolution which contain the ROI of the palmprint of the right hand. Fig. 6 shows the palmprint image samples of 10 users.

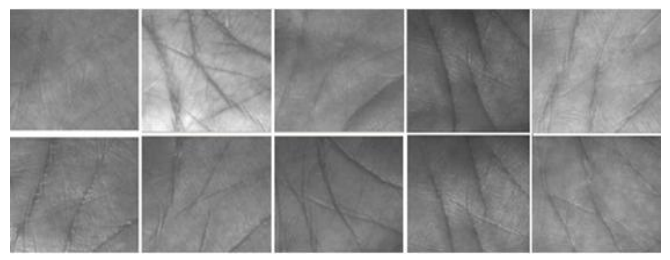

Figure 6. Palmprint image samples

For all feature extraction approaches, we have used the same type of classifier, namely the Support Vector Machines (SVM) with a radial base function. The classmodular classification architecture has been adopted. Table 2 summarizes the performance of the three implemented approaches.

Table 2: Performances recorded for the various proposed approaches

\begin{tabular}{|c|c|c|c|c|c|}
\hline & Approach & $\begin{array}{l}\text { Feature set } \\
\text { size }\end{array}$ & Database & $\begin{array}{l}\text { Recognition } \\
\text { Rate }(\%)\end{array}$ & $\begin{array}{c}\text { Time for features } \\
\text { extraction (s) }\end{array}$ \\
\hline Method in [10] & DWT \& MSE & -- & \multirow{5}{*}{$\begin{array}{l}\text { PolyU 2D, } 400 \\
\text { people, } 8000 \\
\text { images }\end{array}$} & 93 & -- \\
\hline Method in [9] & SIFT \& KNN & -- & & 93.24 & -- \\
\hline Proposed I & Gabor \& SVM & 24 & & 90.57 & 1.62 \\
\hline Proposed II & DWT \& SVM & 5 & & 95.20 & 0.85 \\
\hline Proposed III & CM \& SVM & 4 & & 91.19 & 1.12 \\
\hline
\end{tabular}

The analysis of these results shows the superiority of the DWT in terms of recognition rate compared to other methods. Indeed, the principle of multi-resolution DWT has provided accurate information both in space and frequency on palmprint, which allowed an analysis of the texture at different scales. Based on the results in [10], we also see the effectiveness of our choice of parameters from the wavelet transform, which are the mean and standard deviation of the Approximation
Matrix (AM), the Standard Deviation (SD) of the Horizontal (HD), and the Vertical (VD) and Diagonal Details (DD). Fig 7 shows the distribution of the five parameters resulting from the DWT for a random selection of four users from the PolyU database. We observe the relevance of our features since the overlap areas are much reduced for the mean and standard deviation of the matrix approximation. 


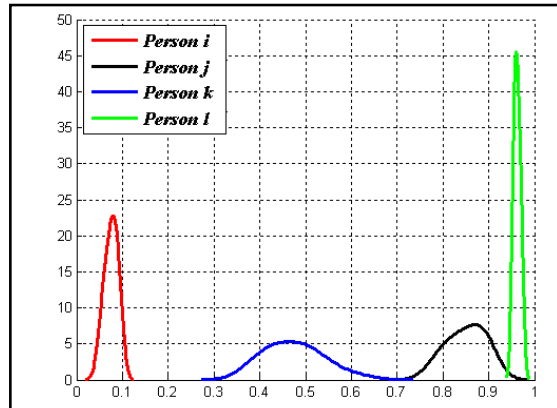

(a)

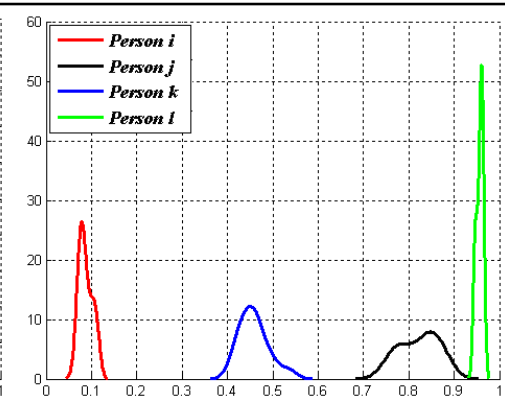

(b)

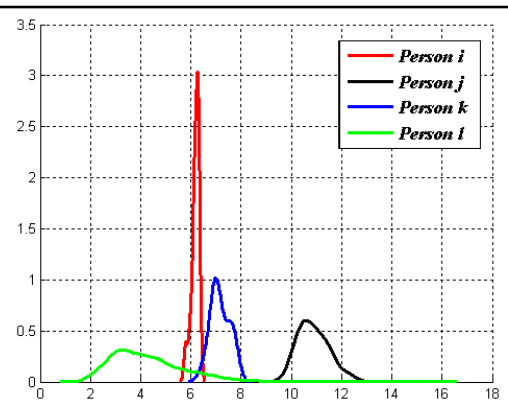

(c)

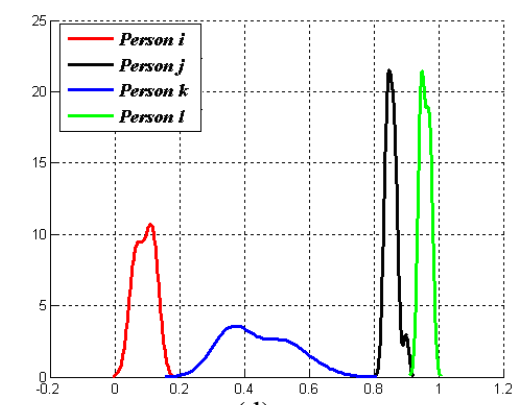

(d)

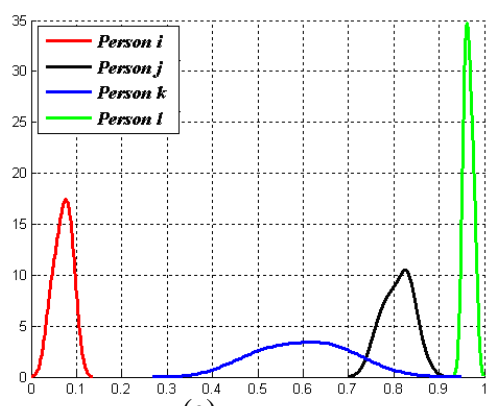

(e)

Figure 7. Distribution of features from the DWT. ((a): mean of the AM. (b): SD of the AM. (c): SD of the HD. (d): SD of the VD. (e): SD of the DD)

In terms of process speed, we have measured, for each approach, the computation time required for the extraction of the characteristics related to one person. From Table 3, we find that the characterization by the wavelet produces a good compromise between the overall system performance and the speed of execution.

The texture analysis by the Gabor filters has led to 24 primitives with a relatively heavy computation time and average performances. This performance can be improved by performing a step of optimization by the genetic algorithms or the PCA to eliminate the redundant frequencies and orientations.

\section{CONCLUSIONS}

In this paper, we have addressed the problems related to the feature extraction from the palmprint in a framework related to the biometric authentication of individuals. Three textural analysis techniques based on the Gabor filters, the Discrete Wavelet Transform and the co-occurrence matrix have been implemented with the same classifier, namely the support vector machines. The results recorded on a database of 8000 images have shown the effectiveness and relevance of the parameters resulting from the wavelet decomposition.

\section{REFERENCES}

[1] A. BenKhalifa, N. Essoukri BenAmara, "Contribution to the Fusion of Biometric Modalities by the Choquet Integral," International Journal of Image, Graphics and Signal Processing, vol.4, no.10, 2012, pp. 1 - 7 .
[2] A. BenKhalifa, N. Essoukri BenAmara, "Exploration of the Choquet integral for the fusion of biometric modalities," The IEEE International Multi-Conference on Systems, Signals \& Devices, 2012, pp. 1 - 6.

[3] A. BenKhalifa, N. Essoukri BenAmara, "Bimodal biometric verification with different fusion levels," The IEEE International Multi-Conference on Systems, Signals \& Devices, 2009, pp. 1 - 6.

[4] A. Kong, D. Zhang, M. Kamel, "A survey of palmprint recognition," Pattern Recognition, Volume 42, Issue 7, 2009, pp. 1408 - 1418.

[5] A. KONG, "Palmprint Identification Based on Generalization of IrisCode," thesis of the University of Waterloo, Canada, 2007.

[6] C.M. Travieso, J.J. Fuertes, J.B. Alonso, "Derivative method for hand palm texture biometric verification," IEEE International Carnahan Conference on Security Technology, 2011, pp. 1 - 5 .

[7] D. Zhang, W.K. Kong, J. You, M. Wong, “On-line palmprint identification," IEEE Transactions on Pattern Analysis and Machine Intelligence, 2003, pp. 1041 - 1050.

[8] H. Masood, M. Mumtaz, M.A.A. Butt, A. Bin Mansoor, S.A. Khan, "Wavelet based palmprint authentication system," International Symposium on Biometrics and Security Technologies, 2008. pp. $1-7$.

[9] H.P. Abeysundera, M.T. Eskil, "Palmprint Verification Using SIFT Majority Voting," 
Computer and Information Sciences II, 2012, pp. $291-297$.

[10] H.B. Kekre, Tanuja, K. Sarode, A.A. Tirodkar, "A study of the efficacy of using Wavelet Transforms for Palm Print Recognition," International Conference on Computing Communication and Applications, 2012, pp. 1 - 6.

[11] H. Imtiaz, S.A. Fattah, "A DCT-based feature extraction algorithm for palm-print recognition," IEEE International Conference on Communication Control and Computing Technologies, 2010, pp. $657-660$.

[12] H. Junlin, Y. Xue, Y. Zhao, J. Lu, "Palmprint recognition based on multiple feature information fusion," International Conference on Signal Processing, 2008, pp. 1449 - 1452.

[13] J. Doublet, "Etude de nouveaux caractères biométriques de la main dans un contexte Télécom," thèse de l'université de CAEN/BasseNormandie, 2006.

[14] K.N. Kumar, "An efficient occlusion invariant Palmprint based verification system," thesis of the University of technology, India, 2009.

[15] K.P. Shashikala, P. Ashwin, K.B. Raja, "Palmprint identification based on fusion of PCA and DTCWT features," International Conference on Image Information Processing, 2011, pp. 1 - 6.

[16] L. Rzouga, A. BenKhalifa, N. Essoukri BenAmara, "Authentification multimodale d'un individu par le visage, l'empreinte digitale et la paume de la main," CRATT'2012, Le Colloque de Recherche Appliquée et de Transfert de Technologie, 2012, pp. $1-6$.

[17] L. Hengjian, L. Wang, "Palmprint recognition using dual-tree complex wavelet transform and compressed sensing," International Conference on Measurement, Information and Control, 2012, pp. $563-567$

[18] M. Ali, M. Ghafoor, I.A. Taj, K. Hayat, "Palm Print Recognition Using Oriented Hausdorff Distance Transform," Frontiers of Information Technology, 2011, pp. 85 - 88.

[19] M.O. Rotinwa-Akinbile, A.M. Aibinu, M.J.E. Salami, "Palmprint Recognition Using Principal Lines Characterization," International Conference on Informatics and Computational Intelligence, 2011, pp. $278-282$.

[20] M. Sharkas, I. El-Rube, M.A. Mostafa, "The Contourlet Transform with the Principal Component Analysis for Palmprint Recognition," International Conference on Computational Intelligence, Communication Systems and Networks, 2010, pp. 262 - 267.
[21] M.P. Dale, M.A. Joshi, N. Gilda, "Texture Based Palmprint Identification Using DCT Features," International Conference on Advances in Pattern Recognition, 2009, pp. 221 - 224.

[22] M. Meiru, Q. Ruan, Y. Shen, "Palmprint Recognition Based on Discriminative Local Binary Patterns Statistic Feature," International Conference on Signal Acquisition and Processing, 2010, pp. 193 - 197.

[23] N. Saini, A. Sinha, "A Palmprint Recognition System Based on Gabor Wigner Transform as Feature Extraction Technique," International Conference on Hand-Based Biometrics, 2011, pp. 1 -5 .

[24] N. Luo, Z. Guo, G. Wu, C. Song, L. Zhou, "Localglobal-based palmprint verification," International Conference on Advanced Computer Control, 2011, pp. 543 - 547.

[25] N. Saini, A. Sinha, "A Palmprint Recognition System Based on Gabor Wigner Transform as Feature Extraction Technique," International Conference on Hand-Based Biometrics, 2011, pp. 1 -5 .

[26] S.K. Panigrahy, "A Secure Template Generation Scheme for Palmprint Recognition Systems,' Master of Technology, university of Technology Rourkela, India, 2008.

[27] S. Shekhar, B.S. Kumar, S. Ramesh, "Robust approach for palm (Roi) extraction in palmprint recognition system," IEEE International Conference on Engineering Education: Innovative Practices and Future Trends, 2012, pp. 1 - 6.

[28] S.M. Prasad, V.K. Govindan, P.S. Sathidevi, "Palmprint authentication using fusion of wavelet based representations," World Congress on Nature \& Biologically Inspired Computing, 2009, pp. 520 -525 .

[29] S. Ribaric, M. Lopar, "Palmprint recognition based on local Haralick features," IEEE Mediterranean Electrotechnical Conference, 2012, pp. 657 - 660.

[30] T.C. Cook, "the development of automated Palmprint identification using major flexion creases," thesis of the University of Wolverhampton, UK, 2012

[31] V.S. Vieira, J.M. Salomão, "Use of wavelet transforms and neural networks for identifying individuals through extracted features of the palm hand," Biosignals and Biorobotics Conference, 2011, pp. 1 - 5.

[32] W. Yuping, J. Zhang, M. Meng, "The study of identification algorithm based on palmprint algebraic features," International Conference on Electrical and Control Engineering, 2011, pp. 3274 - 3277. 
[33] W. Li, D. Zhang, and Z. Xu. "Palmprint identification by Fourier transform," International Journal of Pattern Analysis and Artificial Intelligence, 2002, pp. 417 - 432.

[34] Y. Wang, Q. Ruan, "Kernel fisher discriminant analysis for palmprint recognition," International Conference Pattern Recognition, 2004, pp. 457 460 ,

[35] Z. Liang, W. Taiqing, W. Shengjin, D. Xiaoqing, "DSW feature based Hidden Marcov Model: An application on object identification," International Conference of Soft Computing and Pattern Recognition, 2011, pp. 502 - 506.

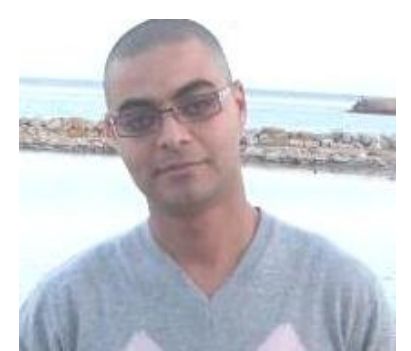

Anouar Ben Khalifa, received the Diploma of Electrical Engineering in 2005 from National Engineering School of Monastir - Tunisia and the Diploma of master in 2007 form National Engineering School of Tunis - Tunisia. $\mathrm{He}$ is currently a D. student and assistant in National Engineering School of Sousse. His research interests are pattern recognition, biometrics and information fusion.

Lamia Rzouga received the Diploma of Industrial Electronic Engineering in 2012 from National Engineering School of Sousse- Tunisia. She is currently a phD. student in National Engineering School of Monastir. Her research interests are biometrics and watermarking.

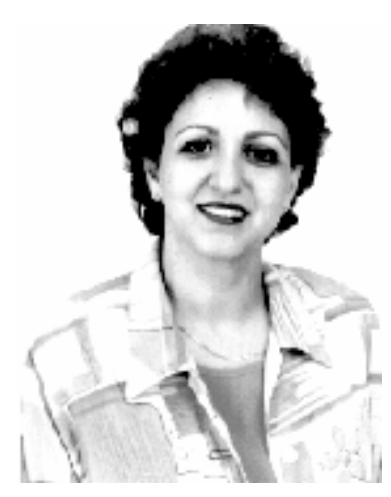

Najoua Essoukri

BenAmara received the B.Sc., M.S., Ph.D. and HDR degrees in Electrical Engineering, Signal Processing, System Analysis and Pattern Recognition from the National School of Engineers of Tunis, University El Manar, Tunisia, in 1985,1986 1999, 2004 respectively.

From 1985 to 1989 , she was a researcher at the Regional Institute of Informatics Sciences and Telecommunications, Tunis, Tunisia. In September 1989 she joined the Electrical Engineering Department of the National School of Engineers of Monastir, University of Monastir, Tunisia, as an assistant professor. She becomes a senior lecturer in July 2004 and a Professor in October 2009 in Electrical Engineering at the National School of Engineers of Sousse-ENISo, University of Sousse, Tunisia. Since July 2008, she is the Director of the ENISo. Her research interests include mainly pattern recognition applied to Arabic documents, ancient image processing, compression, watermarking, segmentation, biometric and the use of stochastic models and hybrid approaches in the above domains. 